\title{
Recovery of Platinum Catalyst and Polymer Electrolyte from Used Small Fuel Cells by Particle Separation Technology
}

\author{
Tatsuya Oki, Takenobu Katsumata, Kazuyuki Hashimoto* and Mikio Kobayashi \\ National Institute of Advanced Industrial Science and Technology, Tsukuba 305-8569, Japan
}

\begin{abstract}
There are several conventional methods for recycling the membrane electrode assemblies (MEA) found in fuel cells. There are chemical techniques that deal with MEAs in bulk, including a method that dissolves the electrolyte film and a method that recovers the catalyst as a residue after incinerating it harmlessly. There are also physical techniques where the electrodes are detached from the MEA one by one. The former approach has a tendency to ignore the recovery of electrolyte film. Moreover, consideration should be given to the fact that the environmental load increases because it is a high-temperature and high-pressure technique that uses a large quantity of chemicals. The target of the latter approach is mainly on-vehicle fuel cells, and in many cases it is unsuitable for the mass processing of the MEAs on fuel cells used in mobile equipment, which are smaller than the above cells. A physical method that detaches the electrodes in organic solvents, which was an early development, offers a promising way to recover both electrodes and electrolyte films, and it can be used to deal with many small MEAs simultaneously. However, it has been pointed out that it is difficult to reuse the recovered electrolyte films, which are deformed and degraded when this method is used. In this study, the individual recovery of electrodes, which include a platinum group metal (PGM) catalyst, and the electrolyte films from MEAs on fuel cells used in mobile equipment, has been studied by using a milder organic solvent so that the deformation and deterioration of the electrolyte film are minimized. [doi:10.2320/matertrans.M-M2009812]
\end{abstract}

(Received December 4, 2008; Accepted March 31, 2009; Published June 10, 2009)

Keywords: fuel cell, membrane electrode assembly, recycling, platinum group metals, electrolyte film, ethanol

\section{Introduction}

The demand from consumers to be able to use mobile devices such as mobile phones and laptop PCs for long periods has increased as device performance has improved. Because the energy density of the current lithium-ion rechargeable battery has almost reached its theoretical limit, the operating time per charge cannot be extended if our aim is to reduce battery weight. As one solution to this problem, small fuel cells have been proposed for mobile devices. By using methanol as a fuel, they are expected to be able to operate for long periods without being charged. ${ }^{1)}$ In stacks of polymer electrolyte fuel cells, MEAs and separators are installed alternately. An MEA consists of electrolyte film, two electrode sheets made of carbon particles carrying PGM catalysts, and two diffusion layers such as carbon papers, which bind these electrolyte films and electrode sheets. Perfluorosulfonic acid films represented by Nafion ${ }^{\circledR}$ are used as the electrolyte films and a diluted film is also used in the electrode binder. The electrodes are formed as sheets with a thickness of several tens of microns, and are made from micron order carbon particles carrying PGM catalysts around $10 \mathrm{~nm}$ in size. When the MEAs are heated, hydrofluoric acid is generated for the recovery of PGM, since fluororesin is used both in the electrolyte films and in the binder of the MEAs. So, pyrometallurgy in a large-scale smelter and concentration by incineration in a small-scale institution cannot be easily carried out. In addition, if PGM is directly dissolved from the MEAs via a leaching process using aqua regia, it would be difficult to leach all the PGM because the fluorine polymer that coats the electrodes would hinder the reaction. Furthermore, the recovery of the fluoride polymer

*Present address: Tokyo Metropolitan Tama Kodaira Public Health Center, Kodaira 187-0002, Japan membrane has to be considered when MEAs are recycled because their cost per MEA is currently as expensive as PGM. For the above-mentioned reasons it is first necessary to separate the PGM from the electrolyte films in MEAs when recycling MEAs.

There are two kinds of techniques for recovering electrolyte films or electrode materials from MEAs. There are chemical methods such as dissolution or incineration, ${ }^{2-7)}$ and there are physical methods such as solid-solid separation. ${ }^{8-10)}$ One representative chemical method is as follows. First the electrolyte films are dissolved in solvents such as alcohol under high pressure in an autoclave in an ambient temperature to $270^{\circ} \mathrm{C}$ range. Then, insoluble PGMs are recovered from the electrode materials. ${ }^{2)}$ The dissolved electrolyte films are used again as materials for electrolyte films or as binder. Another chemical method involves dissolving electrolyte films with oxidation by feeding the MEAs into a reactive fluid consisting of water and oxygen in supercritical states at a high temperature of $400-600^{\circ} \mathrm{C}$ and a high pressure of 23-30 $\mathrm{MPa}^{3)}$ A method for fixing hydrogen fluoride gas generated by the concentration of PGM by using incineration is a moderate and efficient method. ${ }^{4)}$ With this method, the MEAs are ground and mixed with powdered alkali metals, and the harmful gas that is generated by incinerating the mixtures is fixed to solid fluorine components. After that, although PGM can be leached from the burned residue with aqua regia, the components of the electrolyte films are not recovered. With one of the physical methods, electrode materials are detached by using water-soluble adhesion sheets, and the electrolyte film is reused after being recovered through dissolution. ${ }^{8)}$ With the dry ice particle injection method, ${ }^{9)}$ dry ice particles of $1-50 \mu \mathrm{m}$ in size are injected onto electrolyte films or electrodes, which are on the surface of diffusion layers, and then the electrode materials are broken and detached by this impact. The detachment 
efficiency is improved because the electrode materials become fragile after being cooled with dry ice. With this approach, just the electrode materials are recovered because the dry ice vaporizes after the impact. However, these methods would be unsuitable for recycling small MEAs since the electrolyte films are dealt with individually. By contrast, the methods that induce electrode detachment by soaking in liquid can also be applied to small MEAs. ${ }^{10)}$ When an MEA is soaked for around $10 \mathrm{~min}$ in methanol (or a similar solvent), the electrolyte films swell because the methanol substitutes for the water contained in the electrolyte films. Furthermore, the electrolyte films and the electrodes can be separated easily because the binder between them dissolves. Then, the separated electrolyte films are washed with hydrogen peroxide solution after the methanol (or similar) substitution by soaking them in water. However, there is an indication that it is difficult to reuse electrolyte films recovered by this method because the film is deformed and creased after this process. ${ }^{2)}$ In addition to this, methanol, toluene, xylene, and acetone are defined as the second-class organic solvent in the Occupational Safety and Health Act. And, for these solvents, the occupational exposure limits are prescribed as 50 $200 \mathrm{ppm}$, and the toxicity by oral is also provided. ${ }^{11)}$ Lately, although Basf catalysts LLC reported a method which used ethanol to separate electrolyte films and electrodes, the degree of the effect is not identified and the electrolyte films are recycled to raw materials by a chemical method. ${ }^{12)}$ Therefore, the devised methods currently have both merits and demerits, and there is still room for improvement as mentioned above.

This study mainly deals with small fuel cells for use in portable devices, and the investigation focuses on the solidsolid separation of PGM catalysts from fluorine-based polymer films. The method that promotes the detachment of the electrode by soaking the MEA in liquid was adopted as the basic approach, and a way to enable the physical recovery of both PGM and the electrolyte films by using ethanol of a milder solvent, which is not specified the above toxicity, ${ }^{11)}$ was investigated.

\section{Experimental Methods}

\subsection{Samples}

MEAs fabricated by EIWA Corporation were used as samples in this study. The basic characteristics of the MEA are shown in Fig. 1. The MEA consists of five layers: a diffusion layer, a positive electrode, an electrolyte film, a negative electrode, and a diffusion layer. Nafion $117^{\circledR}$ (DuPont) $(80 \mathrm{~mm} \times 80 \mathrm{~mm})$ was used as the electrolyte film. The electrode area was $50 \mathrm{~mm} \times 50 \mathrm{~mm}$. The average thicknesses of 10 points on the electrolyte film and the five-layers electrode were 171 and $620 \mu \mathrm{m}$, respectively, when measured with a digital micrometer (Mitutoyo MDC25M). When the MEA was handled in air, the carbon paper could be peeled easily from the electrolyte film by bending the carbon paper. However, the carbon paper could not be peeled off by turning it horizontally. When the carbon paper was peeled off by bending it, equal amounts of the electrode materials remained on the carbon paper side and the electrolyte film side.

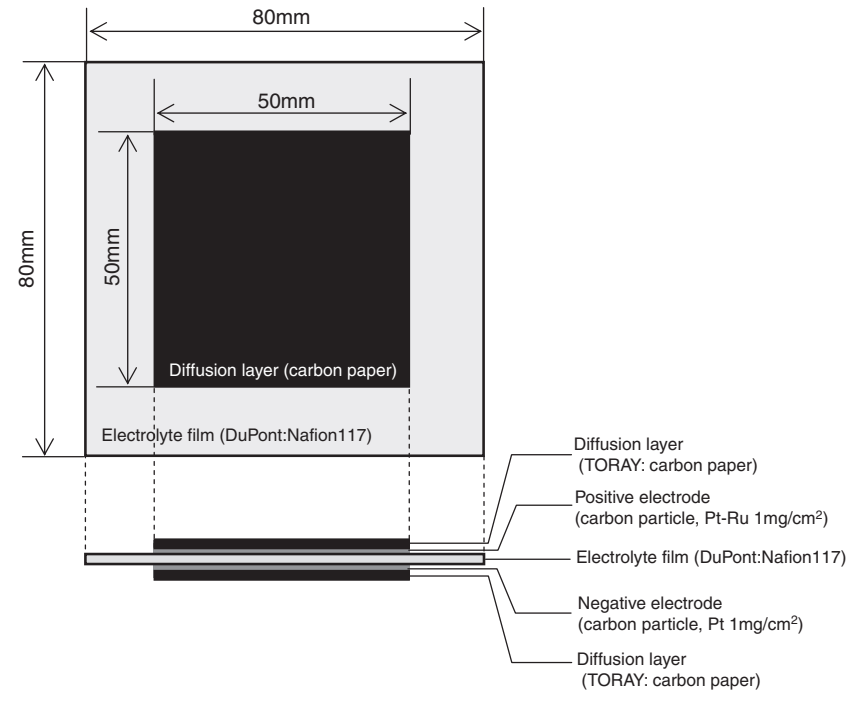

Fig. 1 Schematic diagram of a MEA (5 layers) sample.

\subsection{Experimental methods}

\subsubsection{Electrolyte film behavior with soaking}

To examine the change in the thickness of the electrolyte films with soaking, the samples were cut into $10 \mathrm{~mm} \times$ $10 \mathrm{~mm}$ pieces and placed on a laboratory dish with the crosssection facing upwards, and the dish was placed under a stereo reflecting microscope connected to a video camera. Then, the electrolyte films were soaked in undiluted ethanol $(100 \%)$ solution or $50 \%, 25 \%, 10 \%$ ethanol diluted with deionized water or deionized water. The states of the crosssections of the electrolyte films were recorded with a video camera for $50 \mathrm{~min}$ from the beginning of soaking, and their thicknesses were measured from the obtained digital images. To examine the change in the thicknesses of the electrolyte films during the entire solvent soaking-water soaking-drying process, the electrolyte films were soaked in deionized water for $90 \mathrm{~min}$ after being soaked in the solvents mentioned above for $10 \mathrm{~min}$. They were then dried in a desiccator for more than 20 hours. The thicknesses of the electrolyte films were measured by using digital images recorded with a video camera after the films had been soaked in a solvent for $10 \mathrm{~min}$, in water for $90 \mathrm{~min}$, and after they had been dried.

\subsubsection{Peeling tests for carbon papers}

Stirring, ultrasonic wave irradiation, and stirring + ultrasonic wave irradiation tests were carried out to determine their effectiveness in peeling the carbon paper diffusion layers from the MEAs. In the stirring tests, the MEA samples were placed in a $200 \mathrm{ml}$ water tank containing obstructive boards. The tank was filled with undiluted ethanol (100\%) or $50 \%, 25 \%, 10 \%$ ethanol diluted with deionized water or deionized water (mentioned above), and then the time until the carbon paper peeled off was measured while the mixture was stirred at $111.7 \mathrm{~s}^{-1}$ with a flat impeller (diameter: $7 \mathrm{~mm}$ ). In ultrasonic wave irradiation tests, the MEA samples were placed in a water tank containing obstructive boards, as in the stirring tests, and this tank was then placed in an ultrasonic cleaner. The time until the carbon paper peeled off was measured under ultrasonic wave irradiation conditions with a frequency of $28 \mathrm{kHz}$ and an output of $100 \mathrm{~W}$. In the stirring + ultrasonic wave irradiation tests, the time until the 


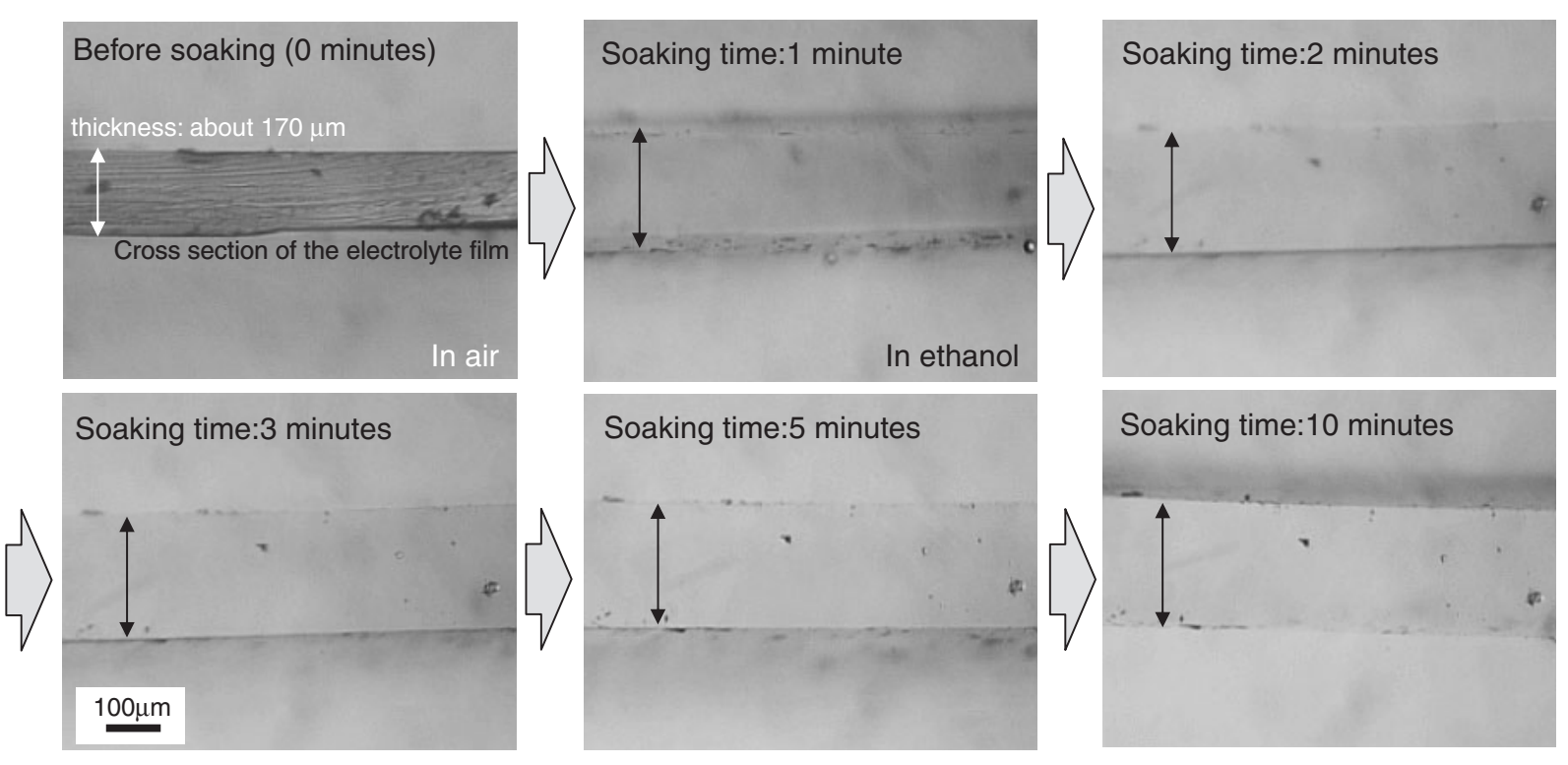

Fig. 2 Change of the cross section of an electrolyte film by soaking in $100 \%$ ethanol with the passage of time.

carbon paper peeled off was measured by stirring the mixture with an impeller and while simultaneously irradiating it with an ultrasonic wave, under the same conditions as those described above. The tests were continued for a maximum of $30 \mathrm{~min}$ if the carbon paper did not peel off.

\subsubsection{Electrode recovery tests}

Stirring, ultrasonic wave irradiation, and stirring + ultrasonic wave irradiation tests were also carried out to examine the detachment of the electrodes from the MEAs. The state at which the carbon paper peeled from the MEA in $10 \%$ of ethanol in the above-mentioned tests, was adopted as the starting condition for the sample. Stirring at $111.7 \mathrm{~s}^{-1}$ and/or ultrasonic wave irradiation at $28 \mathrm{kHz}$ with the methods described above, were carried out for $60 \mathrm{~min}$. After the electrode detachment tests, the electrolyte films were soaked in deionized water for $90 \mathrm{~min}$, and after drying, the total transmittance of the electrolyte films was measured with a transmitting turbidimeter (Nihon-Densyoku NDH-1001DP). After incinerating the original MEA and the electrolyte film, which had peeled from the electrodes, the PGM was extracted with aqua regia, and the PGM concentrations were analyzed with an ICP-AES (Horiba Ultima2). The particle size distributions of the detached electrodes were measured with a laser diffraction particle size analyzer (Beckman Coulter LS230).

\subsubsection{Electrolyte film performance tests}

The I-V curves of five-layers MEAs (amount of platinum catalyst: $1 \mathrm{mg} / \mathrm{cm}^{2}$ ) made of regenerated and brand new electrolyte films were investigated. The tests for the I-V curves were carried out at FC Development Co., Ltd. using a gas supply device (FC Development: Generation Kit) and a load device (Kikusui Electronics: KFM2030 impedance meter). The measurement conditions were as follows: cell temperature: $80^{\circ} \mathrm{C}$, anode fuel: $1 \mathrm{~mol} / 1$ methanol, anode flow: $2.5 \mathrm{ml} / \mathrm{min}$, cathode fuel: air, air flow: $800 \mathrm{~cm}^{3} / \mathrm{min}$ (SCCM), anode line temperature: $40^{\circ} \mathrm{C}$, cathode line temperature: $80^{\circ} \mathrm{C}$. The current was increased in $0.25 \mathrm{~A}(10 \mathrm{~mA} /$ $\mathrm{cm}^{2}$ ) steps and the current maintenance time for the measurements was $30 \mathrm{~s}$.

\section{Results and Discussion}

\subsection{Reaction of electrolyte films during ethanol soaking}

With the methods that promote the detachment of the electrode by soaking in liquid, ${ }^{7)}$ the detachment of the electrode particles is accelerated by soaking the MEA in several kinds of organic solvents, of which methanol is a typical example. Although the new method described here was developed based on this technique, this study focused on using ethanol, which is a mild organic solvent, and on soaking at an ambient temperature, taking cost, environmental load, and the deterioration of the electrode materials into consideration. When an MEA in a dry state was soaked in ethanol, its electrolyte film area grew slightly larger and its thickness increased around 1.5 times. To study the thickness variation of the electrolyte films that were soaked in ethanol in detail, the variation over time was observed by soaking them in undiluted ethanol, in dilute ethanol at several concentrations, and in deionized water. Microscopic images of cross-sections of the electrolyte film that was soaked in undiluted ethanol are shown as example results in Fig. 2, and the variation in the ratio of thickness with the passage of time, when the thickness before soaking was set at 1 , is shown in Fig. 3. In every case the electrolyte films began to swell soon after they had been soaked. The thickness reached its maximum value within one minute when deionized water, $10 \%$ ethanol, and $25 \%$ ethanol were used. The thickness of the electrolyte films that were soaked in $50 \%$ ethanol and undiluted ethanol increased to more than $95 \%$ of their maximum values within the first minute, and reached to their maximum values in around $3 \mathrm{~min}$. The degree to which the thickness of the electrolyte film increased was proportional to the ethanol concentration. The thickness of a film that was soaked in deionized water increased 1.1 times, and for $10 \%$ and $25 \%$ ethanol the thickness increased 1.2 times, for $50 \%$ ethanol it increased 1.55 times, and for undiluted ethanol it increased 1.69 times. In addition, although this is difficult to see in Fig. 2, which is a partial image, the electrolyte film curved with swelling. This appears to result from the fact 


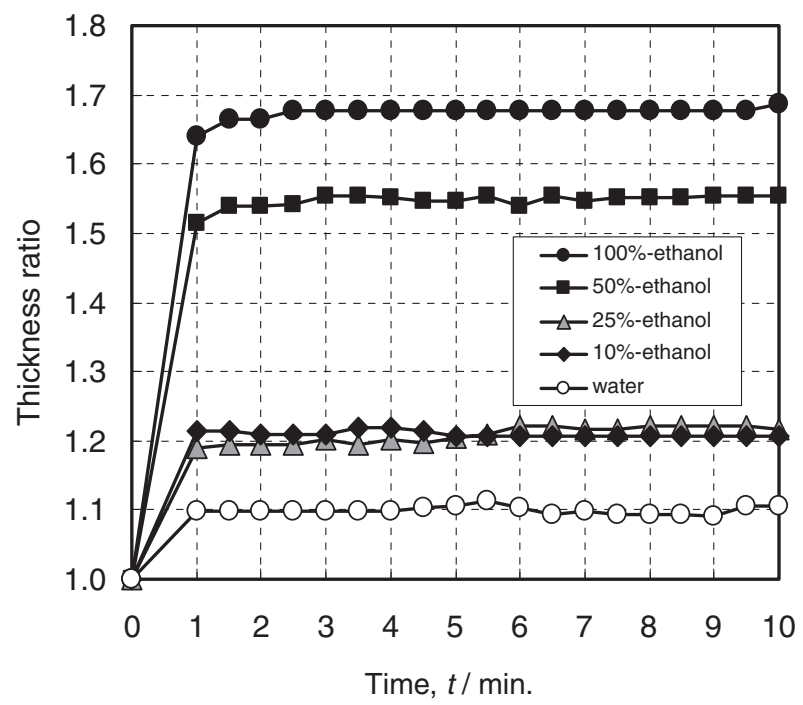

Fig. 3 Change of the thickness ratio of the electrolyte film by soaking in ethanol with the passage of time.

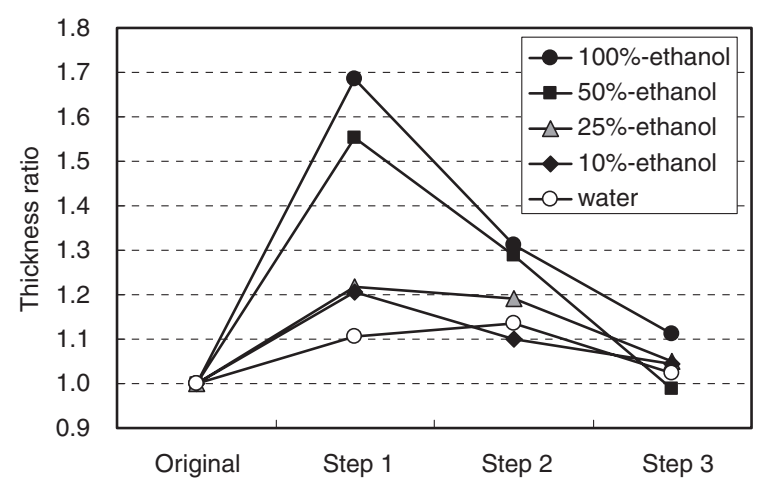

Step 1:Soaking step (After soaking in water or ethanol for 10min.) Step 2:Displacement step (After soaking in water for $90 \mathrm{~min}$.) Step 3:Drying step (After drying for 24 hours.)

Fig. 4 Change of the thickness ratio of electrolyte films by the ethanol soaking-water displacement-drying process.

that the swelling speeds of the two sides of the electrolyte film differed slightly. It was observed that the degree of the curve was especially large with the undiluted ethanol solution, where a sudden change in its thickness occurred quickly. The soaking was continued for $50 \mathrm{~min}$, but there no further change in thickness was observed after $10 \mathrm{~min}$.

Next, electrolyte films that had been soaked in ethanol or deionized water were soaked in deionized water for $90 \mathrm{~min}$ and then dried. The changes in the thickness ratio of the electrolyte films in each step are shown in Fig. 4. Although the thicknesses of the electrolyte films changed with the ethanol concentration in the soaking step, they largely recovered their original thicknesses of $160-180 \mu \mathrm{m}$ after soaking in water (step 2) and drying (step 3). However, although it can restore an electrolyte film to its original thickness, the water displacement-drying process cannot restore the curve of the film. Therefore, the ratio of the thickness of the film and the height of the film in the appearance, when the original and treated (soaked-displaceddried) films were placed on a flat plate, was measured to express the degree of this curve quantitatively. Figure 5

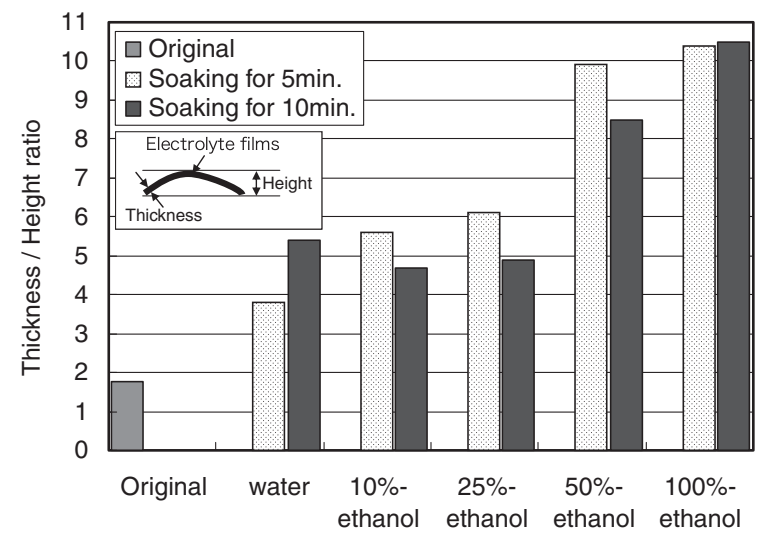

Fig. 5 Thickness/height ratio at each soaking condition after the drying process.

shows the thickness/height ratio of the electrolyte films for the original film, after $5 \mathrm{~min}$ soaking, and after $10 \mathrm{~min}$ soaking. The thickness/height ratio of the original film was 1.75 (the average value for 12 samples). Since the thickness of the original film was $171 \mu \mathrm{m}$, the apparent height would also be $171 \mu \mathrm{m}$ if the electrolyte film was flat and the film was in full contact with the flat plate. However, the electrolyte film did not come into complete contact with the flat plate, because the film was deformed. The apparent film height was 1.75 times the film thickness, namely $299 \mu \mathrm{m}$, and this means that there was a maximum gap of $128 \mu \mathrm{m}$ between the film and the plane surface. For the films that had been treated by soaking in water or in $10 \%$ ethanol or in $25 \%$ ethanol, followed by displacement in water, and drying, the thickness/height ratio exhibited an approximately fivefold increase. For the films that had been treated by soaking in ethanol in the $50 \%$ to undiluted range, the thickness/height ratio increased about $8-10$ times. The degree of the ratio increase was sometimes opposite after soaking for 5 and $10 \mathrm{~min}$, which indicates that the change in the curve was irregular compared with the change in thickness. However, the ratio became constant when the ethanol concentration was lower than $25 \%$. Further observations revealed that the curve shapes could be grouped to two types as in Figs. 6(b) and $6(\mathrm{c})$. After being soaked in ethanol with a concentration of $25 \%$ or lower, the film curved gently in the same direction, and the paper started to form a cylindrical shape as shown in Fig. 6(b). When pushed from the top and bottom onto a flat plane, it would easily recover its flat state. On the other hand, when the ethanol concentration was $50 \%$ or higher, the paper curved along one side of the $\mathrm{x}-\mathrm{y}$ axis forming a saddle shape, as shown in Fig. 6(c). Once the film adopts such a form, it will never recover its flat state even if it is pressed from the top and bottom, and it would be difficult to reuse it as a new electrolyte film even if its chemical function was acceptable. Although some papers ${ }^{13-15)}$ have reported about the chemical function change of electrolyte films by its swelling, investigation on the mechanism of $3 \mathrm{D}$ deformation by swelling such as the above results have not been reported as far as the authors know. The above results show that it is important to conduct the separation process with ethanol concentrations of less than $25 \%$, to minimize any deformation of the electrolyte film. 


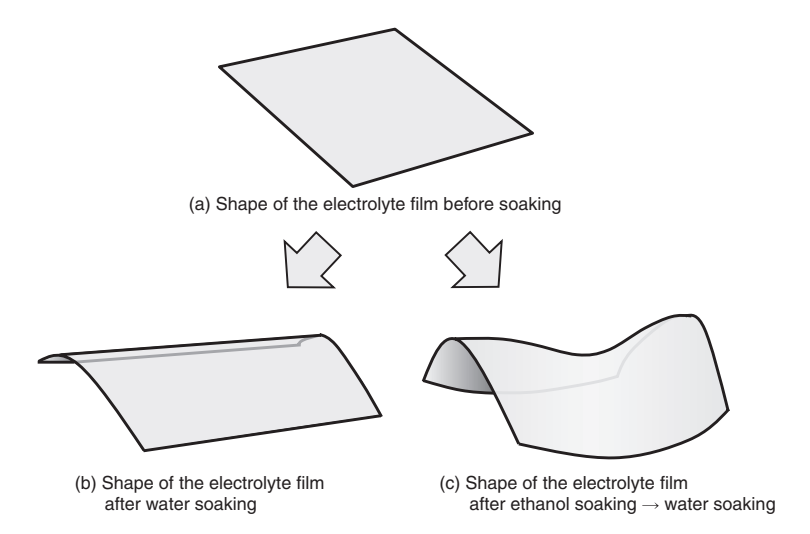

Fig. 6 Change of shape of the electrolyte film after water and ethanol soaking.

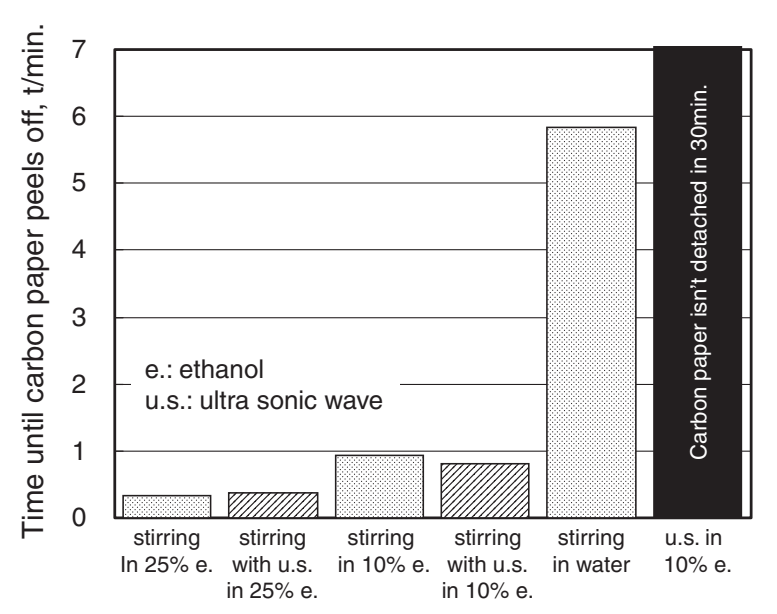

Fig. 7 The time necessary for peeling off the carbon paper.

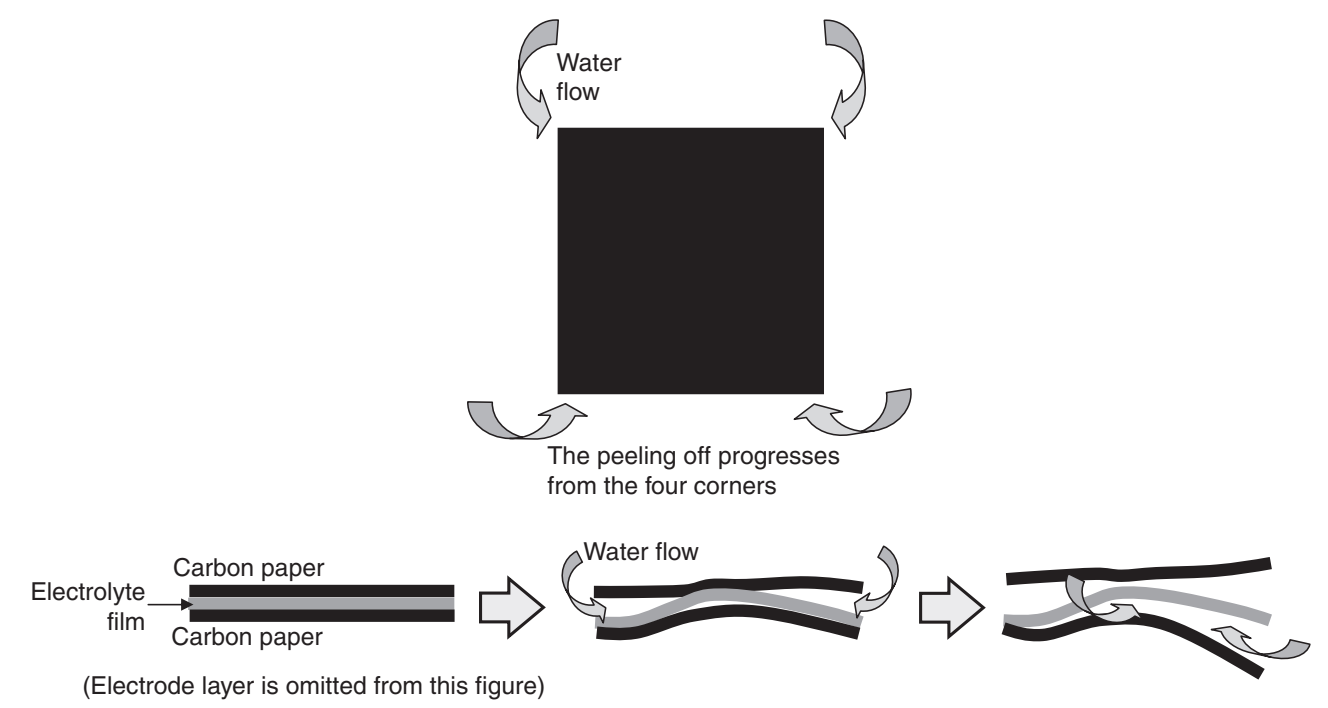

Fig. 8 The image of peeling off of the diffused layer (carbon paper).

\subsection{Detachment of the electrode by soaking in ethanol 3.2.1 Peeling off the carbon paper}

It is first necessary to peel off the carbon paper, which is a diffused layer, in order to detach the electrode by soaking the MEA in ethanol. So, the conditions required for rapidly peeling off the carbon paper were investigated. The results are shown in Fig. 7. It took 6 min. for the carbon paper to peel off in stirred water which was slow. However, it took only $50 \mathrm{~s}$ and $20 \mathrm{~s}$, respectively, in $10 \%$ and $25 \%$ ethanol. In a preliminary experiment, where a $45 \mathrm{~mm}$ impeller was used to stir the sample at $8.3 \mathrm{~s}^{-1}$ in $10 \%$ ethanol, the carbon paper did not peel off even after $15 \mathrm{~min}$. The results were almost the same even when the sample was irradiated with an ultrasonic wave. With ultrasonic wave irradiation alone, the carbon paper did not peel off even after $30 \mathrm{~min}$. These results made it clear that the carbon paper is peeled off by soaking the sample in ethanol and that shearing is achieved by stirring at high speed. It also became clear that the irradiation of an ultrasonic wave during the stirring does not interfere with the peeling process, but that peeling is not enhanced by its use. Observations of the process showed that the peeling proceeds as shown in Fig. 8 in almost every case. That is, first the carbon paper is peeled from the four corners of the MEA, and then water flows in. Next, the paper peels off completely from the center, assisted by the effect of the deformation of the electrolyte film.

\subsubsection{Recovery of electrode}

The same amount of carbon particle holding PGM, which constitutes an electrode, attaches to the electrolyte film surface and to the carbon paper surface when the carbon paper peels off. Part of the electrode disperses into the liquid phase during the peeling process, but a considerable amount remains on the electrolyte film. To realize both the reuse of the electrolyte film and the recovery of the PGM of the electrode, which is the aim of this study, the electrode must be detached completely from the electrolyte film. Therefore, electrode detachment was investigated by using the tests described above, that is, stirring, ultrasonic wave irradiation, and stirring + ultrasonic wave irradiation.

With a view to using a solvent whose concentration is as low as possible, the state after the carbon paper had been peeled off in a $10 \%$ ethanol in the tests described above was adopted as the starting condition for the sample. After the detachment treatments, the electrolyte films were soaked in deionized water for $90 \mathrm{~min}$ and then dried, and the total transmittance was measured with a transmitting turbidimeter. 


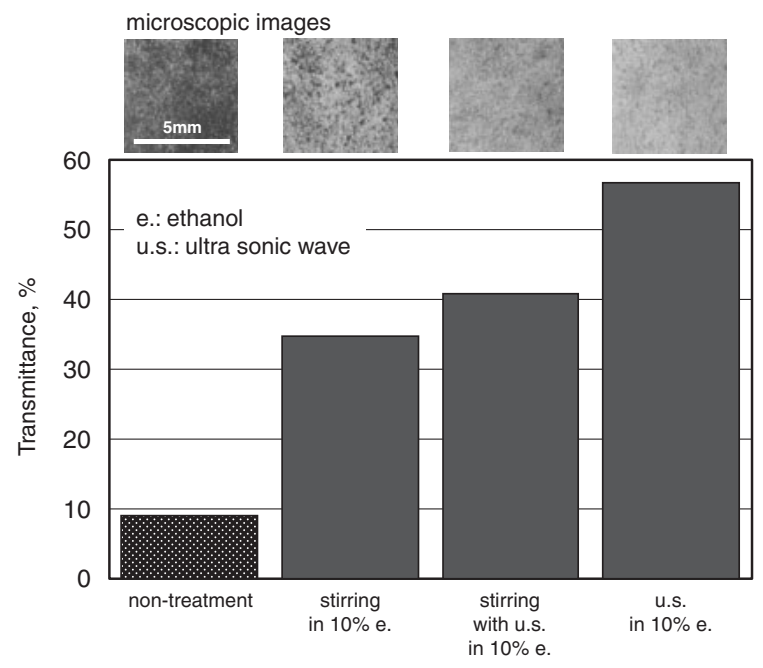

Fig. 9 Transmittance of the electrolyte films after detachment treatments.

The results are shown in Fig. 9. The electrolyte film was almost transparent and the carbon holding PGM was black, so a higher transmittance means that the enhancement of detachment is relatively high. Here, non-treatment means the condition immediately after the carbon paper has been peeled off. After every treatment the transmittance becomes larger than without any treatment, which indicates that the electrode detachment is induced by the treatments. However, unlike when peeling off the carbon paper, the tendency of the effect was stirring $<$ stirring + ultrasonic wave irradiation $<$ ultrasonic wave irradiation. This reveals that ultrasonic wave irradiation is more effective than stirring as regards detaching the electrodes and that the effect of ultrasonic wave irradiation is hindered by stirring. When only ultrasonic wave irradiation was employed, almost all of the electrode detached from the electrolyte film, although a few black spots remained. After incinerating the original MEA and the electrolyte film from which the electrode was detached, the PGM was extracted with aqua regia, and the PGM concentration was analyzed with an ICP-AES. The results showed that $9.7 \%$ of the PGM remained on the electrolyte film, which means that $90.3 \%$ of PGM was recovered.

The carbon particles of the electrode that were detached from the electrolyte film were a few to approximately ten microns in size. However, if the particles are broken by the irradiation of an ultrasonic wave so that their size is less than a few microns, the burden on the solid-liquid separation imposed after this process would become heavier. So, to examine whether or not the sizes of the detached electrodes differ with changes in ultrasonic wave irradiation time, the particle size distribution of the detached electrodes was measured with a laser diffraction particle size analyzer. The results are shown in Fig. 10. After ten min. of ultrasonic wave irradiation, the particle sizes ranged from $30-150 \mu \mathrm{m}$, and the $50 \%$ diameter in volume $\mathrm{D}_{50}$ was $88 \mu \mathrm{m}$. This indicates that the particles were detached as aggregates. $\mathrm{D}_{50}$ was 105 and $108 \mu \mathrm{m}$ after 30 and 90 min of ultrasonic wave irradiation, respectively, which indicates that the particle size becomes larger over time. These results suggest that the

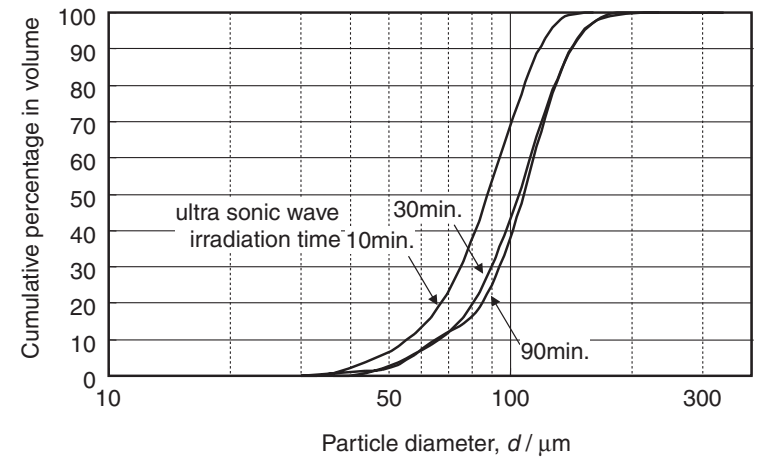

Fig. 10 Particle size distribution of the electrode (carbon particles holding PGM) detached with ultra sonic wave irradiation.

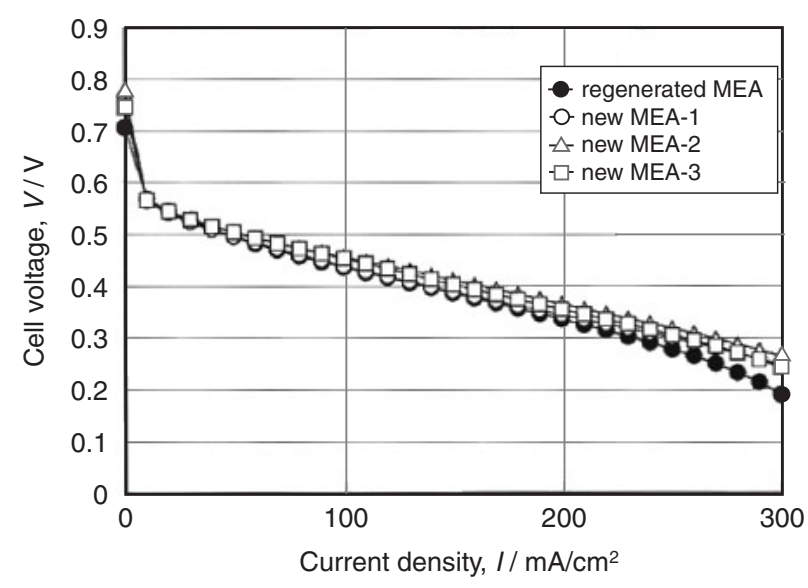

Fig. 11 I-V curve of the MEA made from regenerated electrolyte film.

aggregated particles that had detached from the electrolyte film did not become smaller even when exposed to an ultrasonic wave, and consequently the size of the particles that disperse in ethanol becomes progressively larger because larger aggregates will detach from the electrolyte film as the ultrasonic wave irradiation time becomes longer.

\subsubsection{Electrolyte film performance}

The degree to which the performance of a regenerated MEA made from electrolyte films recovered during the above tests differed from that of a brand new MEA was investigated. The I-V curves of a regenerated and brand new electrolyte films are shown in Fig. 11. As seen from this graph, the performance of the regenerated MEA differs little from that of the new one, although the current of the regenerated MEA becomes slightly lower when the voltage is low. Figure 12 compares the output density at $0.4 \mathrm{~V}$, where the electricity generation efficiency is high, on the fuel consumption. Although the value is slightly inferior to the average value for three new MEAs, it is almost the same as that of "new MEA-1", and the MEA regenerated by this recycling method stands comparison with a new one.

The investigations conducted in this study make it clear that the regenerated electrolyte is only slightly deformed, and the performance is as good as a that of a new one, despite the fact that it uses only a $10 \%$ ethanol concentration at ambient temperature, which is a milder condition than that of the conventional methods. 


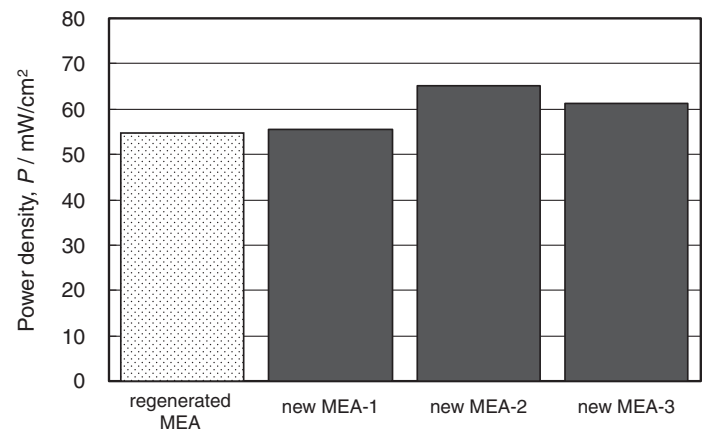

Fig. 12 Power density of the MEA made from regenerated electrolyte film.

\section{Conclusion}

This study investigated a method for recovering electrodes that include a PGM catalyst, and the electrolyte films from the MEAs of fuel cells used in mobile devices by using dilute ethanol so that the deformation and deterioration of the electrolyte films are minimized. The results are summarized as follows.

(1) Ethanol soaking tests showed that the ethanol concentration must be lower than $25 \%$ to minimize the deformation of the electrolyte film. It became clear that the diffused layered carbon paper can be peeled off (the first process with this method) by soaking the sample in ethanol and by the shearing that is realized by stirring at high speed.

It was also made clear that ultrasonic wave irradiation during the stirring did not interfere with the peeling of the carbon paper, but that the peeling would not be enhanced by its use.

(2) Ultrasonic wave irradiation is more effective than stirring as regards detaching the electrode, which is unlike the case when peeling off the carbon paper. It was also shown that the effect of ultrasonic wave irradiation was hindered if stirring was used at the same time. When ultrasonic wave irradiation in $10 \%$ ethanol was employed, $9.7 \%$ of the PGM remained on the electrolyte film, which means that $90.3 \%$ of the PGM was recovered. Since the sizes of the detached carbon particles holding PGM range from $30-150 \mu \mathrm{m}$, it is comparatively easy to recover them.

(3) The I-V curves of MEAs fabricated using recovered electrolyte film and completely new film were compared. Although the value is slightly inferior to the average value for three new films, the performance was equivalent to one of the MEAs made with new electrolyte film. Therefore, it is considered that a regenerated electrolyte film is only slightly deformed, and can be reused with almost the same level of performance as that of a brand new electrolyte film.

\section{REFERENCES}

1) Fuji-Keizai Inc.: Nano-tech kanren shijo kibo doko chosa hokokusho (2006).

2) Japanese patent: JP-A-H11-288732.

3) Japanese patent: JP-A-2003-531964.

4) Japanese patent: JP-A-2004-190092.

5) C. Handley, N. P. Brandon and R. van der Vorst: J. Power Sources 106 (2002) 344-352.

6) H. F. Xu, X. Wang, Z. G. Shao and I. M. Hsing: J. Appl. Electrochem. 32 (2002) 1337-1340.

7) J. Zhao, X. He, J. Tian, C. Wan and C. Jiang: Energ. Conversion Management 48 (2007) 450-453.

8) Japanese patent: JP-A-2005-235511.

9) Japanese patent: JP-A-2006-95367.

10) Japanese patent: JP-A-H8-171922.

11) The Japan society for occupational health: J. Occup. Health 50 (2007) 426-443.

12) United States patent: US 2006/0237034 A1.

13) L. C. haabane, G. Bulvestre, C. Larchet, V. Nikonenko, C. Deslouis and H. Takenouti: J. Membl. Sci. 323 (2008) 167-175.

14) G. J. Elfring and H. Struchtrup: J. Membl. Sci. 315 (2008) 125-132.

15) A. Sacca, A. Carbone, R. Pedicini, G. Portale, L. D’Ilario, A. Longo, A. Martorana and E. Passalacqua: J. Membl. Sci. 278 (2006) 105-113. 